\title{
An intronic PICALM polymorphism, rs588076, is associated with allelic expression of a PICALM isoform
}

\author{
Ishita Parikh¹, Christopher Medway², Steven Younkin², David W Fardo ${ }^{3}$ and Steven Estus ${ }^{\text {* }}$
}

\begin{abstract}
Background: Although genome wide studies have associated single nucleotide polymorphisms (SNP)s near PICALM with Alzheimer's disease (AD), the mechanism underlying this association is unclear. PICALM is involved in clathrin-mediated endocytosis and modulates $A ß$ clearance in vitro. Comparing allelic expression provides the means to detect cis-acting regulatory polymorphisms. Thus, we evaluated whether PICALM showed allele expression imbalance (AEI) and whether this imbalance was associated with the AD-associated polymorphism, rs3851179.

Results: We measured PICALM allelic expression in 42 human brain samples by using next-generation sequencing. Overall, PICALM demonstrated equal allelic expression with no detectable influence by rs3851179. A single sample demonstrated robust global PICALM allelic expression imbalance (AEI), i.e., each of the measured isoforms showed AEl. Moreover, the PICALM isoform lacking exons 18 and 19 (D18-19 PICALM) showed significant AEl in a subset of individuals. Sequencing these individuals and subsequent genotyping revealed that rs588076, located in PICALM intron 17, was robustly associated with this imbalance in D18-19 PICALM allelic expression $\left(p=9.54 \times 10^{-5}\right)$. This polymorphism has been associated previously with systolic blood pressure response to calcium channel blocking agents. To evaluate whether this polymorphism was associated with AD, we genotyped 3269 individuals and found that rs 588076 was modestly associated with AD. However, when both the primary AD SNP rs3851179 was added to the logistic regression model, only rs3851179 was significantly associated with AD.

Conclusions: PICALM expression shows no evidence of AEI associated with rs3851179. Robust global AEI was detected in one sample, suggesting the existence of a rare SNP that strongly modulates PICALM expression. AEI was detected for the D18-19 PICALM isoform, and rs588076 was associated with this AEl pattern. Conditional on rs3851179, rs588076 was not associated with AD risk, suggesting that D18-19 PICALM is not critical in AD. In summary, this analysis of PICALM allelic expression provides novel insights into the genetics of PICALM expression and $A D$ risk.
\end{abstract}

Keywords: PICALM, Alzheimer's disease, Next-generation sequencing, Allelic expression imbalance, Single nucleotide polymorphism

\section{Background}

Phosphatidylinositol binding clathrin assembly protein (PICALM) facilitates clathrin-mediated endocytosis. PICALM binds phosphatidylinositol 4,5- bisphosphate $\left(\mathrm{PIP}_{2}\right)$, adaptor protein $2\left(\mathrm{AP}_{2}\right)$ and clathrin to mediate endocytic clathrin coated vesicle formation at the plasma membrane. Although PICALM is ubiquitously expressed,

\footnotetext{
* Correspondence: Steve.estus@uky.edu

${ }^{1}$ Departments of Physiology, Sanders-Brown Center on Aging, University of Kentucky, 800 S. Limestone St., Lexington, KY 40536, USA

Full list of author information is available at the end of the article
}

PICALM expression is more pronounced in microvessels $[1,2]$. Previous studies have shown PICALM co-localizes with APP and modulates amyloid beta (Aß) generation [3-5]. Accumulation of $A ß$ deposits is a hallmark of Alzheimer's Disease (AD) pathology.

Genome wide association studies in multiple cohorts have identified single nucleotide polymorphisms (SNP)s near the PICALM gene as significantly associated with $\mathrm{AD}$ risk [6-10]. Studies were first conducted with Caucasian populations and then independently verified in several although not all Asian populations [11-15]. These 
studies report that the rs3851179 A allele reduces AD risk with an odds ratio of 0.88 [6]. This SNP is located approximately $80 \mathrm{~kb} \mathrm{5'}$ of PICALM.

Understanding how rs3851179 alters PICALM to impact AD risk may lead to novel insights into AD mechanisms and potential treatments. Since rs3851179 is not in linkage disequilibrium (LD) with a SNP that alters a PICALM amino acid $\left(\mathrm{r}^{2}<0.1\right)$, we hypothesize that rs3851179 is associated with changes in mRNA transcription or processing. Allelic expression imbalance (AEI), which is an expression difference between allelic transcripts within an individual, has been used to detect cis-regulatory effects [16-20]. Here, we performed an AEI analysis by comparing allelic expression through the use of two exonic SNPs, rs76719109 and rs592297, in $\mathrm{AD}$ and non-AD brain samples. These studies included 35 samples that were heterozygous for rs76719109 and 19 samples that were heterozygous for rs592297. While PICALM expression did not show AEI overall, one individual showed robust PICALM AEI, with an allelic ratio of 0.76. Additionally, significant AEI was detected for the PICALM isoform lacking exons 18 and 19 (D18-19 PICALM). Sequencing and additional genotyping established that rs588076 was robustly associated with this AEI pattern. Interestingly, rs588076 has been associated with blood pressure response to ${ }^{\mathrm{Ca}++}$ channel blocking agents [21]. We discuss these overall results in the context that genetic regulation of PICALM isoforms relative to AD risk is highly complex with further work necessary to elucidate the mechanisms modulating genetic risk.

\section{Results}

To detect the presence of regulatory cis-acting SNPs in human brain samples, we measured allelic ratios in cDNA from reverse transcribed mRNA. Heterozygosity for an exonic "reporter" SNP provides the means to compare the expression of one allele with another allele within an individual. Our criteria for reporter SNPs for AEI analysis is that the SNPs are present in exons and have a minor allele frequency (MAF) greater than $15 \%$, which allows for sufficient sample numbers for analysis. Only two PICALM SNPs satisfied these criteria, rs76719109 and rs592297 (Figure 1). Rs76719109 has a MAF of 0.44 and resides within exon 17; PCR amplification from exon 17-20 allowed us to measure AEI for total PICALM as well as PICALM splice variants lacking exon 18 or exons 18-19 (Figure 1a). Rs592297 has a MAF of 0.20 and resides in exon 5. PCR amplification from exon 5-6 produced a single PCR product for cDNA (Figure 1b). The AEI assay was validated in two ways. First, we tested the linearity of the assay by generating a cDNA standard curve consisting of five different rs76719109 T:G ratios (Figure 2). Our input T:G ratios ranged from 1:4 to 4:1. We found a robust linear relationship between input and observed T:G ratios. Second, we applied our experimental approach to genomic DNA (gDNA), which represented a positive control with an expected "allelic" ratio of 1.0. Rs76719109 and rs592297 showed gDNA ratios of $1.01 \pm 0.03$ (mean \pm $\mathrm{SD}, \mathrm{n}=35)$ and $0.96 \pm 0.05$ (mean $\pm \mathrm{SD}, \mathrm{n}=19$ ), respectively (Figure 3). Hence, this AEI assay appears robust for detecting and quantifying variations in allelic expression.

To evaluate whether the AD-associated SNP rs3851179 was associated with unequal allelic PICALM expression, we performed AEI analysis with rs76719109 and rs592297on a total of 54 samples. Twelve of these 54 samples were heterozygous for both SNPs. Hence, we analyzed PICALM for AEI in a total of 42 unique individual samples. This effort analyzed 4.2 million sequences for rs76719109 and 1.4 million sequences for rs592297. If rs3851179 modulated total PICALM expression, we expected to see significant AEI in individuals heterozygous for rs3851179, but not in individuals homozygous for rs3851179. When we analyzed the results for the exon 17 SNP, rs76719109, significant AEI was observed in only a single sample, termed AD40 (see below). To evaluate whether a subtle difference in allelic expression may be present and associated with rs3851179, we compared the mean allelic expression between the rs3851179 homozygous and heterozygous groups. This approach did
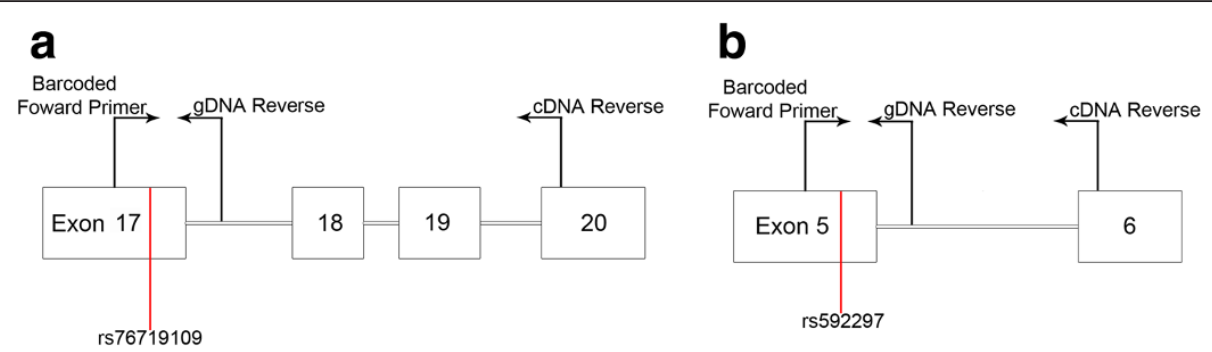

Figure 1 Rs76719109 and rs592297 AEI assays. a) For the exon 17 SNP rs76719109, a barcoded forward primer was positioned in exon 17, and a reverse primer was positioned in intron 17 (genomic samples) or exon 20 (cDNA samples). b) For the exon 5 SNP rs592297 assay, a barcoded forward primer was positioned in exon 5, and a reverse primer was positioned in intron 5 (genomic samples) or exon 6 (cDNA samples). 


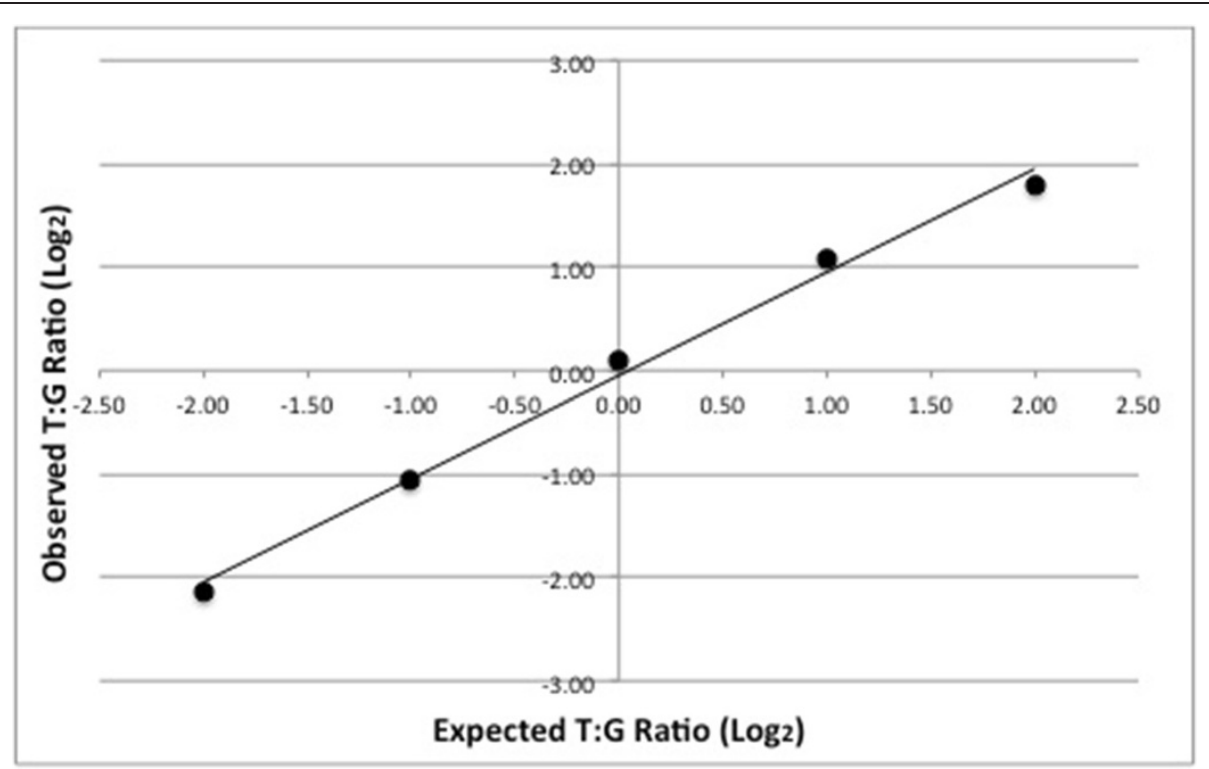

Figure 2 Linearity of allelic expression assay. Different proportions of rs76719109 T and G homozygous cDNA were mixed to test the linearity of the AEl assay. The T:G ratios were 1:4, 1:2, 1:1, 2:1, and 4:1. An overall linear relationship was found $\left(r^{2}=0.99\right)$. The slope was 0.999 , i.e., the assay detected the $T$ and $\mathrm{G}$ alleles with equal efficiency. The graphs are plotted $\log _{2}$ to avoid compression at the lower ratios and thereby better visualize the data [22].

not discern a significant difference between the two groups, i.e., the allelic ratios for rs3851179 homozygous and heterozygous groups was $0.94 \pm 0.08(n=17)$ and $0.97 \pm 0.06(n=$ 18), respectively (Figure 4a, $\mathrm{p}=0.24$, t-test). Hence, rs3851179 did not appear associated with PICALM AEI. To confirm this finding and extend the analysis to additional samples, we analyzed allelic expression by using the exon 5 SNP, rs592297. Significant AEI was not observed in any sample, noting that the sample with the significant AEI result from the rs76719109 analysis was homozygous for rs592297 and not suitable for evaluation. When the allelic ratios were analyzed by t-test to evaluate whether rs3851179 was associated with allelic expression, the findings confirmed that rs3851179 was not associated with robust $\mathrm{AEI}$; the allelic ratios were $0.93 \pm 0.05(n=4)$ for rs3851179 homozygotes and 1.01 $\pm 0.07(\mathrm{n}=15)$ for heterozygotes (Figure $4 \mathrm{~b}, \mathrm{p}=0.06$ ). Hence, we found that the total PICALM allelic ratio for each person was remarkably consistent with both reporter SNPs; rs3851179 is not associated with overall allelic PICALM expression.

Interestingly, cDNA from the individual termed AD40 showed significant unequal allelic mRNA expression with a T:G ratio of 0.76 (Figure 4a, Additional file 1: Table S1).

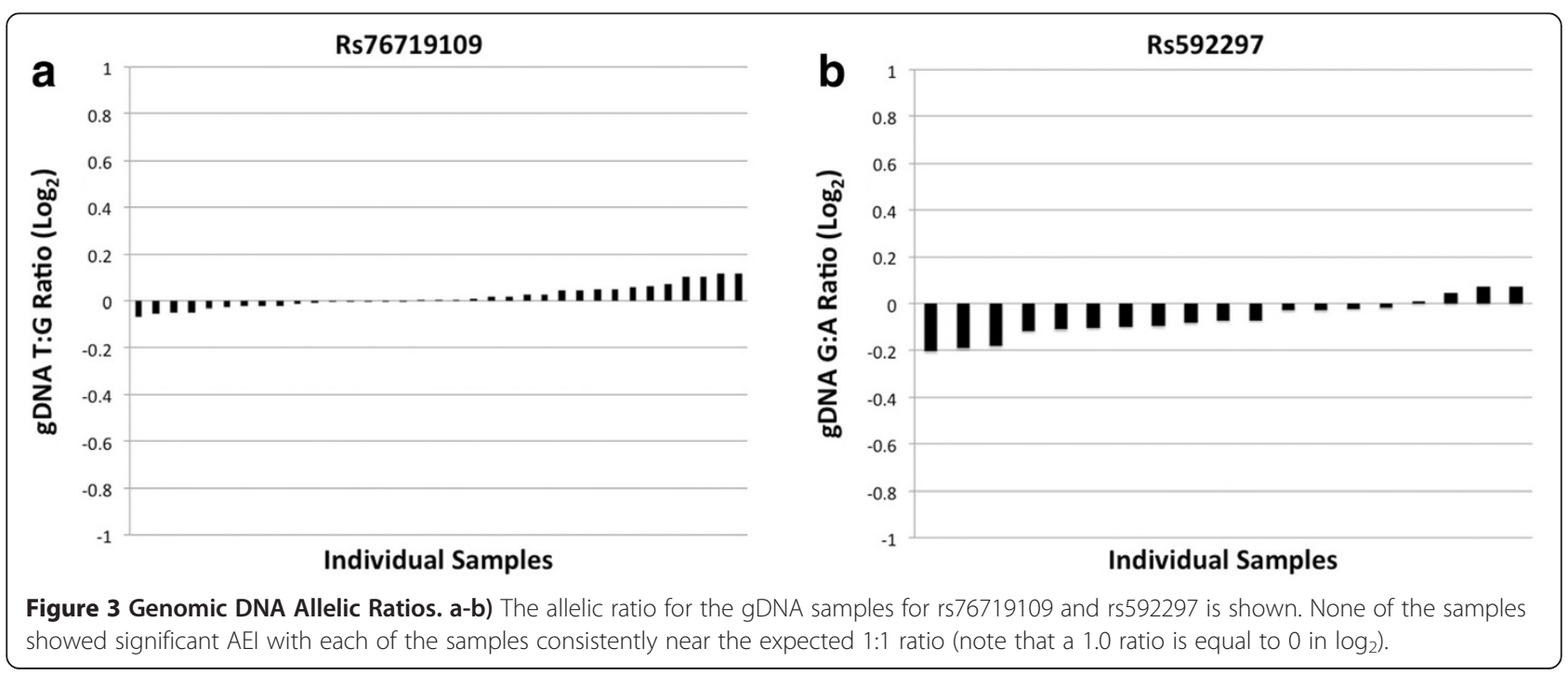




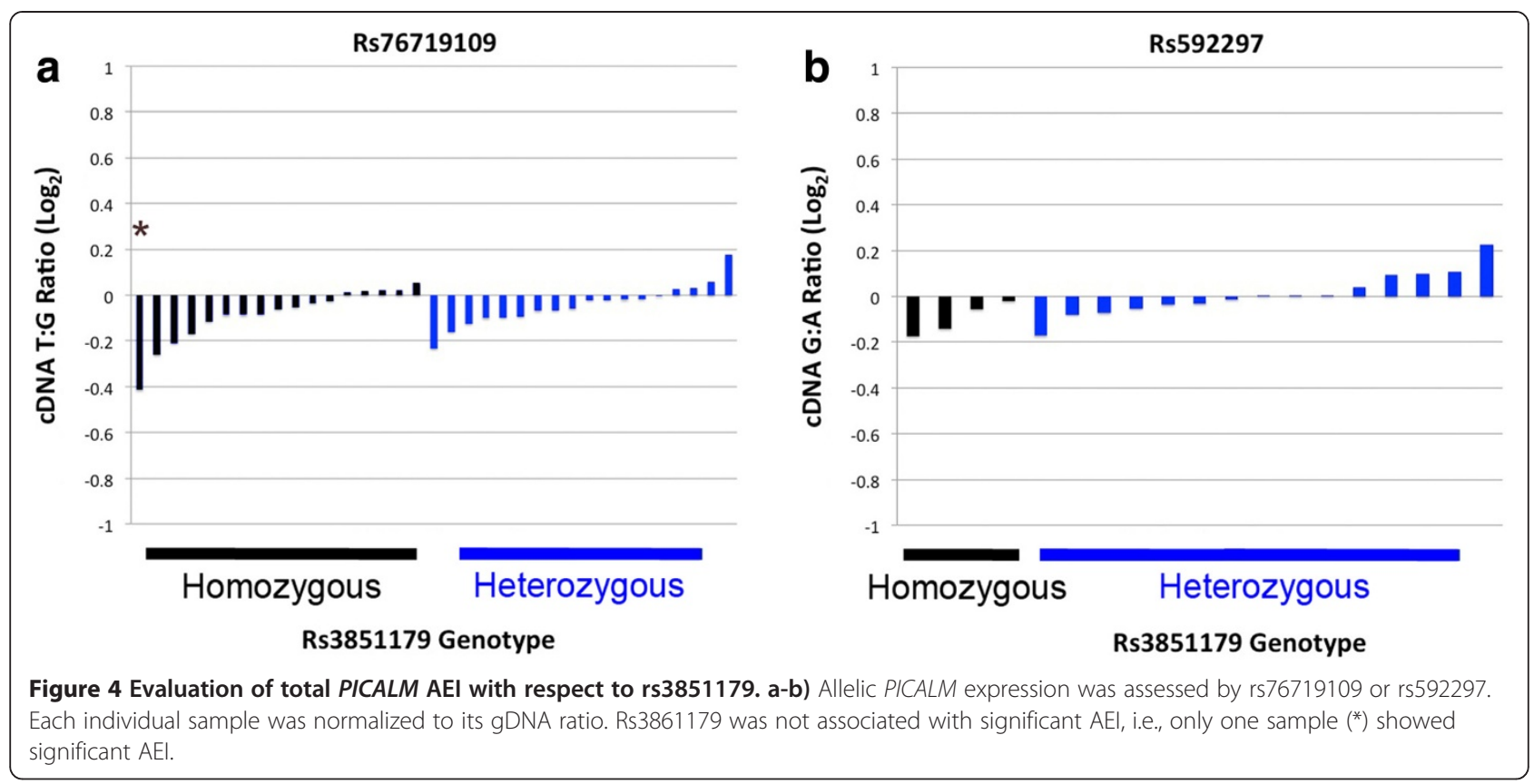

This ratio is based on a total of four experiments that detected a total of 68141 copies of the T allele and 88400 copies of the $\mathrm{G}$ allele. This AEI was not due to genomic normalization as the genomic T:G ratio was 1.01. These data are based upon the rs76719109 SNP because this individual was homozygous for rs592297. A similar result was obtained when each of four separate replicates were analyzed individually, i.e., when each replicate was analyzed individually, the T:G ratio was $0.73 \pm 0.04$ (mean \pm SD). Hence, significant AEI was observed in a single individual among the 42 unique samples.

Having considered overall PICALM allelic expression, we proceeded to apply this AEI analysis to PICALM isoforms. The exon 17 to exon 20 PCR amplicons captured three different PICALM isoforms because exons 18 and 19 are variably spliced. These isoforms were termed full length PICALM (contains exons 18-19), D18-PICALM (lacked exon 18) or D18-19 PICALM (lacked both exons 18 and 19). We analyzed each of these isoforms for the presence of AEI as a function of rs3851179 heterozygosity. For the full length PICALM and D18-PICALM isoforms, significant AEI was observed only in the AD40 sample. To evaluate whether a subtle difference in allelic expression may be present, we compared the allelic expression of the full length PICALM isoform between the rs3851179 homozygous and heterozygous groups by using a t-test. However, no difference was observed as the average T:G ratio in the rs3851179 homozygous and heterozygous groups was $0.93 \pm 0.07(\mathrm{n}=17)$ and $0.93 \pm$ $0.06(\mathrm{n}=18)$, respectively (Figure $5 \mathrm{a}, \mathrm{p}=0.81)$. Likewise, for the D18-PICALM isoform, the rs3851179 homozygous and heterozygous groups showed mean T:G ratios of $0.95 \pm 0.09(n=17)$ and $1.01 \pm 0.12(n=18)$, respectively (Figure $5 \mathrm{~b}, \mathrm{p}=$ value $=0.13$ ). When we evaluated the D18-19 PICALM isoform, significant AEI was detected in multiple samples (Figure 5c). We analyzed these results in two ways. First, we compared the frequency of samples with significant AEI between the rs3851179 homozygous and heterozygous individuals by using a Fisher's exact test; a significant difference between groups was not detected (Figure $5 \mathrm{c}, \mathrm{p}=0.44)$. Second, we compared the mean T:G ratio between rs3851179 homozygous and heterozygous individuals by using a t-test. However, the rs3851179 homozygous and heterozygous individuals showed similar values that did not achieve significance, i.e., $1.19 \pm$ 0.16 and $1.11 \pm 0.22$, respectively $(\mathrm{p}=0.08)$. Hence, rs3851179 heterozygosity was not associated with AEI for these PICALM isoforms.

We considered the subset of samples that showed significant AEI further. Among these, AD40 showed significant AEI for each of the isoforms, with the D18, D18-19 and full length PICALM isoforms having allelic T:G ratios of $0.77,0.53$ and 0.77 , respectively. As noted above, this finding is consistent with the hypothesis that a rare SNP acts to alter global PICALM allelic expression in this individual. Additionally, we noted that the D18-19 PICALM isoform showed significant AEI for multiple individuals (Figure 5c). In these eight individuals, the rs76719109T allele was expressed more than the $\mathrm{G}$ allele with the ratio ranging from 1.30 to 1.48 . This is in contrast to AD40 where the $\mathrm{T}$ allele was expressed less than $\mathrm{G}$ allele at a ratio of 0.53 (Table 1 ).

Since the D18-19 PICALM isoform but not overall PICALM showed AEI in multiple samples, we hypothesized 


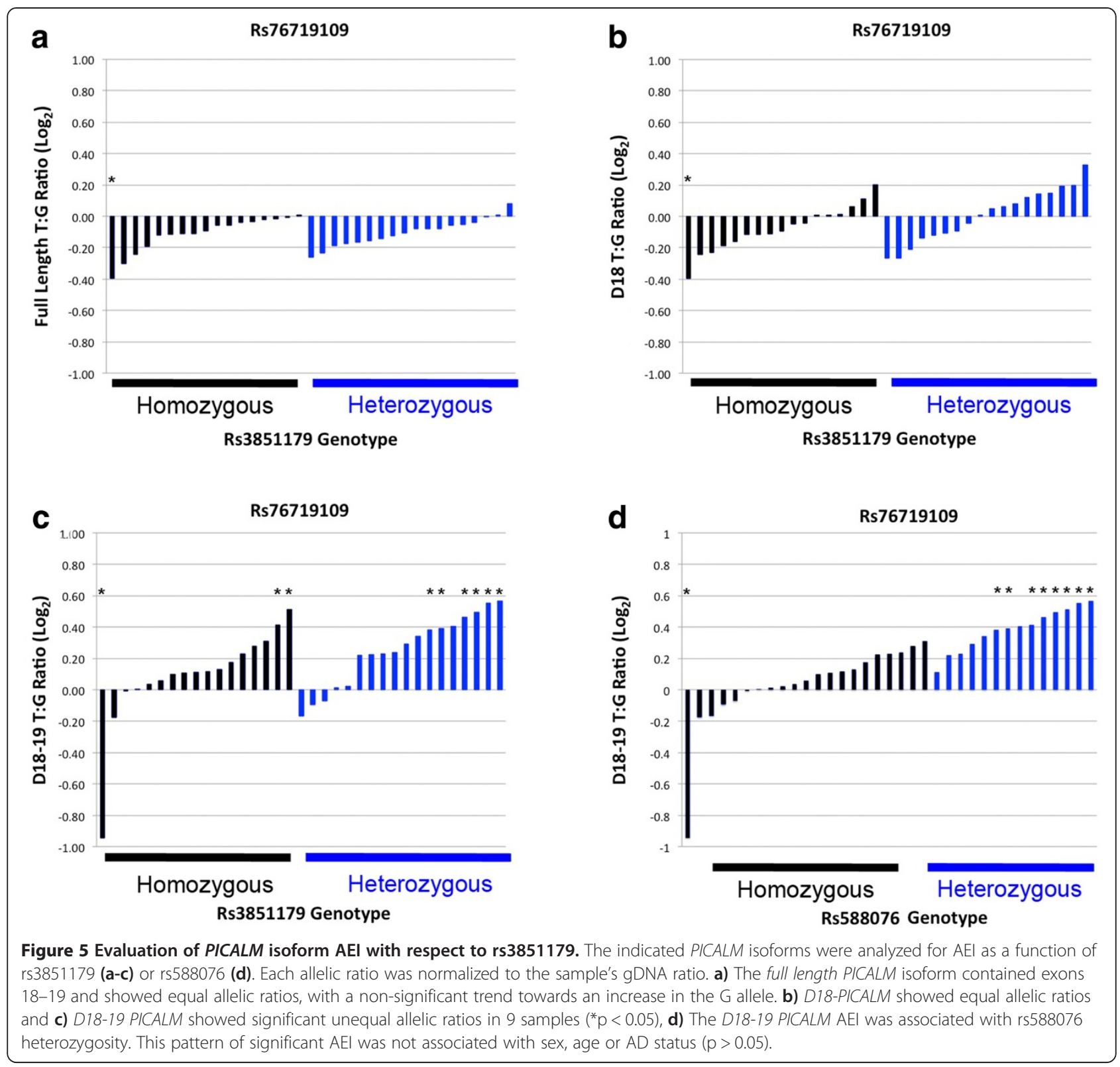

Table 1 D18-19 PICALM shows significant AEI in nine samples

\begin{tabular}{cccc}
\hline Sample & D18-19 T:G Counts & Ratio (Normalized to genomic ratio) & P-Value \\
\hline C01 & $2337: 1793$ & 1.30 & $8.66 \times 10^{-3}$ \\
C11 & $13415: 9508$ & 1.47 & $7.91 \times 10^{-34}$ \\
C20 & $745: 523$ & 1.43 & $2.78 \times 10^{-3}$ \\
AD33 & $6136: 4162$ & 1.48 & $7.06 \times 10^{-25}$ \\
AD43 & $1743: 1321$ & 1.31 & $9.47 \times 10^{-3}$ \\
AD50 & $1529: 1080$ & 1.41 & $3.05 \times 10^{-5}$ \\
AD51 & $5530: 4243$ & 1.33 & $5.27 \times 10^{-5}$ \\
AD54 & $8893: 6548$ & 1.38 & $2.64 \times 10^{-14}$ \\
AD40 & $2203: 4103$ & 0.53 & $1.96 \times 10^{-65}$
\end{tabular}

These D18-19 PICALM T:G allelic counts are the summation of three separate runs. AD samples are designated by an AD prefix while non-AD samples are designated with a $C$ prefix. 
that a local SNP influences this splice pattern. To identify this SNP, we sequenced 6400 bp of genomic DNA between exons 17-20 in C11 and AD33, the two individuals showing the highest AEI ratio (Table 1). We identified several SNPs that were heterozygous in these samples including rs588076, rs645299, and rs618629. The MAF for these SNPs in CEU range from 20-31\% (Table 2). Additionally, these SNPs are in strong LD with each other (Table 2). Rs588076 and rs618629 reside in intron 17 while rs645299 is within intron 18. We genotyped all samples for rs588076. The frequency of samples showing significant AEI was significantly associated with rs588076 heterozygosity (Figure $5 \mathrm{~d}, \mathrm{p}=9.54 \times 10^{-5}$, Fisher's exact test). We interpret these results as strongly suggesting that rs588076, or a SNP in strong LD with rs588076, is functional by modulating PICALM exon 18-19 splicing.

Since rs588076 is associated with D18-19 PICALM AEI and rs3851179 has been robustly associated with $\mathrm{AD}$, we evaluated the extent that rs588076 is associated with AD risk. We evaluated $1789 \mathrm{AD}$ and 2529 non-AD individuals from the Mayo Clinic cohort. Rs588076 was significantly associated with AD risk when analyzed in a logistic regression model (odds ratio $=0.8413,95 \%$ confidence intervals (0.7151-0.9898), $\mathrm{p}=0.0372$ ). However, when rs3851179 was added to this logistic regression model, only rs3851179 was significantly associated with AD (Table 3). Haplotypic analysis confirmed that the haplotype containing both rs588076 and rs3851179 report the same association with $\mathrm{AD}$ as the haplotype containing only rs3851179 (data not shown). Hence, rs588076 is associated with D18-19 PICALM AEI but not AD risk.

\section{Discussion}

The primary findings of this report include (i) overall PICALM expression shows no evidence of global AEI even when parsed by AD-associated SNPs, (ii) robust global AEI was detected in one sample, suggesting the existence of a rare SNP that strongly modulates PICALM expression, and (iii) eight individuals show AEI for the D18-19 PICALM isoform that is associated with rs588076. However, rs588076 was not associated with AD risk when considered in a model that also included rs3851179. In summary, analysis of allelic expression has proved a useful tool for the evaluation of cis-acting regulatory polymorphisms and $\mathrm{AD}$ risk.
A consistent pattern of AEI in overall PICALM expression was not detected. This was unexpected since $\mathrm{Xu}$ et al. reported consistent and robust PICALM AEI [22]. The reason for different results in these two studies is unclear. The studies are similar in that both used rs76719109 as a reporter SNP and similar although not identical PCR primers. The studies differ in that Xu et al. used an Asian population while this report studied Caucasians. One explanation that would account for the difference in the studies was the presence of a confounding SNP in the Asian population in the genomic primer sequence because much of the AEI in Xu et al. was due to correction for imbalance in gDNA [22], although such a SNP has not yet been reported. We previously reported that the AD-associated SNP rs3851179 was associated with a modest difference in PICALM expression when analyzed relative to cell-type specific mRNAs; the minor rs3851179A allele appeared to be expressed modestly higher than the $G$ allele [2]. A similar difference was not observed here. One possible interpretation of these findings is that rs3851179 or its proxy AD SNP acts in a cell-type specific fashion that was discernible in our analysis that included cell-type specific markers. The current AEI study had smaller sample size because only the samples that were heterozygous for rs76719019 or rs592297 were suitable for analysis. However, this would not be expected to affect the AEI results because they rely upon an intra-individual analysis. We interpret these results overall as suggesting that the $\mathrm{AD}$-associated SNP, or its functional proxy, acts in a cell-type specific fashion to modulate PICALM expression. This cell-type specific action was not detectable in this AEI study of mRNA derived from multiple cell types.

The second major finding was that robust AEI was detected for all PICALM isoforms in one individual, arguing for the existence of a rare functional SNP that strongly modulates total PICALM expression. For this individual, the rs76719109G allele was consistently more abundant than the T allele for each PICALM isoform. We hypothesize that $\mathrm{AD} 40$ is unique among the 42 samples in showing AEI because this sample is heterozygous for a causal SNP. If this causal allele is present in the heterozygous state in 1 of 42 people, this SNP has a minor allele frequency of $\sim 1.2 \%$. Although current sequencing studies of the PICALM promoter region have not yet identified candidate functional SNPs for AEI in this sample, these

Table 2 Samples with robust AEI are heterozygous for three SNPs

\begin{tabular}{|c|c|c|c|c|c|}
\hline$\overline{\text { SNP }}$ & MAF in CEU & LD with rs3851179 & LD with rs588076 & LD with rs645299 & LD with rs618629 \\
\hline rs588076 & 0.199 & 0.336 & - & 0.539 & 0.911 \\
\hline rs645299 & 0.306 & 0.665 & 0.539 & - & 0.525 \\
\hline rs618629 & 0.242 & 0.319 & 0.911 & 0.525 & - \\
\hline
\end{tabular}

The samples C11 and AD33 were sequenced from exon 17 through exon 20. The samples were heterozygous for the indicated SNPs. These SNPs are in strong LD [23] with each other and have similar frequencies for the minor allele [24]. 
Table 3 Logistic regression modeling of rs3851179 and/or rs588076 effect(s) on AD

\begin{tabular}{ccccc}
\hline Model & SNP & Odds Ratio & Confidence Interval & P value \\
\hline rs588076 & rs588076 & 0.8413 & $0.7151-0.9898$ & 0.0372 \\
rs3851179 & rs3851179 & 0.7786 & $0.6819-0.8891$ & 0.0002 \\
rs3851179+rs588076 & rs3851179 & 0.7767 & $0.6604-0.9136$ & 0.0023 \\
rs3851179+rs588076 & rs588076 & 1.005 & $0.8238-1.227$ & 0.959 \\
\hline
\end{tabular}

In addition to the indicated SNPs, these models were adjusted for age of onset, APOE alleles, sex and contributing center.

studies are on-going and a SNP that strongly modulates PICALM expression would be expected to be a robust AD risk factor.

The third major finding was that the D18-19 PICALM isoform showed robust AEI. There was a strong skew towards increased expression of the rs76719109T allele. Sequencing identified several candidate SNPs including rs588076, which is 509 bp downstream of exon 17. This SNP was found to be robustly associated with AEI for D18-19 PICALM. There are three possible ways rs588076 could influence D18-19 PICALM splicing efficiency: (i) rs588076 is in high LD with a functional SNP that modulates splicing, (ii) rs588076 and other SNPs influence D18-19 PICALM splicing in a cooperative manner, and/or (iii) rs588076 directly influences splicing. Further studies are necessary to discern among these possibilities.

The biological significance of the rs588076 association with D18-19 PICALM is complex. D18-19 PICALM transcripts account for $1-2 \%$ of total PICALM expression [2]. Thus rs588076 is significantly associated with AEI for a PICALM isoform that is relatively rare in brain. Exons 18 and 19 encode a total of 27 amino acids that are part of the carboxyl terminal region required for clathrin binding and endocytosis [25]. Hence, the protein encoded by D18-19 PICALM is likely to have reduced function [25]. However, rs588076 was not associated with AD risk and did not enhance the logistic regression model for the rs3851179 association with AD. This leads us to conclude that the rs588076 and D18-19 PICALM isoform may be too rare in the brain to influence $A D$ pathogenesis. Interestingly, rs588076 was recently associated with the blood pressure response to ${ }^{\mathrm{Ca}++}$ channel blocking agents [21]. Since rs588076 is associated only with D18-19 PICALM, we speculate that this isoform may be more abundant in other tissues and rs588076 actions upon D18-19 PICALM mediate this systolic blood pressure phenotype.

\section{Conclusion}

In summary, analysis of allelic expression has shown that compelling PICALM AEI was not observed in most brain RNA samples. Strong global AEI was documented in one sample, suggesting the existence of a rare PICALM regulatory SNP. A pattern of AEI was clearly discerned for the
D18-19 PICALM isoform and rs588076 was significantly associated with this pattern. Rs588076 was not associated with AD risk although this SNP has been associated with a blood pressure-related phenotype. Allele-dependent expression studies may provide further insights into additional AD-associated polymorphisms.

\section{Methods}

\section{DNA and RNA extraction from human brain tissue}

The RNA and DNA samples for this study were from deidentified $\mathrm{AD}$ and non-AD human brain anterior cingulate specimens provided by the University of Kentucky AD Center Neuropathology Core and have been described previously $[2,26,27]$. The overall dataset included $30 \mathrm{AD}$ samples (14 male, 16 female) and 30 non-AD samples (15 male, 15 female). The age at death for individuals that were cognitively intact, i.e., non-AD, was $82 \pm 8$ years (mean \pm $\mathrm{SD}, \mathrm{n}=30$ ) while age at death for AD individuals was $82 \pm$ $6(\mathrm{n}=30)$. The average post-mortem interval (PMI) for non-AD individuals was $2.8 \pm 0.9$ hours (mean $\pm \mathrm{SD}, \mathrm{n}=$ 30) while the PMI for AD individuals was similar at $3.4 \pm$ 0.6 hours $(\mathrm{n}=30)$. For the rs76719109 AEI assay, a subset of 35 samples were heterozygous for this SNP and included 18 non-AD ( 9 male, 9 female) and 17 AD (9 male, 8 female). For the rs592297 AEI assay, a total of 19 out of 60 samples were heterozygous, 13 non-AD (7 male, 6 female) and $6 \mathrm{AD}$ (3 male, 3 female). Preparation of gDNA, RNA and cDNA was performed as described in previous studies $[2,26,27]$. Although RNA integrity analyses were not performed prior to reverse transcription, others have demonstrated that for qPCR with short amplicons, normalized expression differences are comparable between samples with moderate RNA degradation and those with high integrity RNA [28]. We recognize that the absence of RNA integrity analysis constitutes a caveat of this study.

\section{Genotyping and sequencing}

DNA samples were genotyped for rs3851179, rs76719109, rs592297 and rs588076 by using unlabeled PCR primers and two allele-specific TaqMan FAM and VIC dye-labeled MGB probes (Pre-designed TaqMan SNP Genotyping Assay, Applied Biosystems) on a real-time PCR machine (Chromo4, MJ Research PTC-200). 


\section{Allelic imbalance assay}

Rs76719109 is in exon 17. For AEI analysis with rs76719109, PICALM was amplified from exon 17 to exon 20 for cDNA and exon 17 to intron 17 for genomic DNA. For rs592297, PICALM was amplified from exon 5 to exon 6 for cDNA and exon 5 to intron 5 for genomic DNA. Exon numbering is according to PICALM005 ENST00000393346 for exons 1-16 and exons 17-21 correspond to the final five exons within PICALM-002 ENST00000532317 in Ensembl, since no single ENSEMBL transcript includes each of the exons the we identify here [29]. The PCR primers included Ion Torrent adapters, individual barcodes and DNA sequence flanking the region of interest (Table 4). Each PCR reaction (50uL) contained 1x PCR buffer, 20 ng cDNA or 100 ng gDNA, 1uM of forward and reverse primer, $0.1 \mathrm{mM} \mathrm{dNTP}$ and 0.5 Units Taq (Platinium Taq, Invitrogen). Cycles consisted of preincubation at $95^{\circ} \mathrm{C}$ for 2 minutes, followed by 28 cycles of $95^{\circ} \mathrm{C}$ for $15 \mathrm{~s}, 60^{\circ} \mathrm{C}$ for $30 \mathrm{~s}$ and $72^{\circ} \mathrm{C}$ for $60 \mathrm{~s}$ followed by incubation at $75^{\circ} \mathrm{C}$ for 7 minutes. To acquire equal representation from each sample, relative amounts of PCR product were quantified by subjecting $10 \mathrm{uL}$ of PCR product to electrophoresis on $7.5 \%$ polyacrylamide gels and SYBR gold staining relative to a Low DNA Mass Ladder (Invitrogen). Approximately $2 \mathrm{ng}$ of each individual's cDNA and gDNA PCR products were pooled, purified using Agencourt AMPure XP and subjected to Ion Torrent sequencing on a Ion Torrent 316 chip (Ion PGM Sequencer).

Data extraction and analysis of allelic mRNA expression Allelic counts were extracted from DNA sequences by using Perlscript in a three-step fashion: (i) sequences corresponding to each sample were separated based on their barcode, (ii) gDNA and cDNA were then separated based on the presence of intronic and exonic sequences, respectively, and (iii) allele counts were obtained by using sequences that bridged the SNP of interest.

\section{Standard curve generation}

One rs76719109 homozygous major (GG) and one homozygous minor (TT) individual was selected based on similar qPCR copy numbers. Five dilutions were prepared with different ratios of each individual's cDNA: 1:4, 1:2, 1:1, 2:1, and 4:1. These samples were PCR amplified and subjected to sequencing as described above.

\section{Statistical analysis}

Analysis of allelic counts was based upon the assumption that transcript read counts follow a Poisson distribution [30]. As such, each allele from the heterozygous SNP was used to define two random variables. Following the rs76719109 example of a G/T SNP, we denote the pair of transcript counts as $G \sim$ Poisson $\left(\lambda_{G}\right)$ and $T \sim$ Poisson $\left(\lambda_{T}\right)$. That is, $\mathrm{G}$ and $\mathrm{T}$ are Poisson-distributed random variables with means $\lambda_{G}$ and $\lambda_{T}$, respectively. It can then be readily shown that for a given pair of realized transcripts counts, $\mathrm{G}=\mathrm{g}$ and $\mathrm{T}=\mathrm{t}$, the transcript count of either allele is binomially distributed with success probability equal to a ratio of component means. That is, $G \mid G+T=g+t \sim \operatorname{Bin}\left(g+t, p=\frac{\lambda_{G}}{\lambda_{G}+\lambda_{T}}\right)$. Testing for AEI then simplifies to an examination of the null hypothesis that the pair of transcript counts comes from the same distribution, i.e., that $\lambda_{G}=\lambda_{T}$ which is equivalent to testing $H_{0}: p=\frac{1}{2}\left[G \mid G+T=g+t \sim \operatorname{Bin}\left(g+t, \frac{1}{2}\right)\right]$. This null hypothesis agrees with the intuition that when the total of transcript counts is known, the number generated from a specific allele is essentially a sequence of independent, equally probable trials. Thus, rejection of this null hypothesis corresponds to AEI.

Measuring transcripts from genomic DNA is one way of correcting for the possibility of differential experimental error between allele transcript counts. Conceptually, one could adapt methods for determining AEI by an appropriate adjustment with the ratio of reads from gDNA as these reads should theoretically come from the same distribution regardless of AEI (Fardo et al., unpublished).

Table 4 PCR primers for rs76719109 and rs592297 AEI assay

\begin{tabular}{|c|c|c|c|}
\hline Assay & DNA & Primer Sense & Primer Sequence \\
\hline \multirow[t]{4}{*}{ rs76719109 } & CDNA & Sense & 5'CCATCTCATCCCTGCGTGTCTCCGACTCAGXxxxxTGGAGTCAACCAGGTGAAAA \\
\hline & CDNA & Anti-sense & 5' CCTCTCTATGGGCAGTCGGTGATTTGGTTGCGTCATTACAGGA \\
\hline & gDNA & Sense & 5'CCATCTCATCCCTGCGTGTCTCCGACTCAGxxxxxTGGAGTCAACCAGGTGAAAA \\
\hline & gDNA & Anti-sense & 5' CCTCTCTATGGGCAGTCGGTGATAGGAGCTIITCAACTCACCA \\
\hline \multirow[t]{4}{*}{ rs592297 } & CDNA & Sense & 5'CCATCTCATCCCTGCGTGTCTCCGACTCAGxxxxxTGAACACAGAAAAACTCCTAAAAA \\
\hline & CDNA & Anti-sense & 5' CCTCTCTATGGGCAGTCGGTGATGGCAGCATTTATTACCCCATT \\
\hline & gDNA & Sense & 5'CCATCTCATCCCTGCGTGTCTCCGACTCAGxxxxxTGAACACAGAAAAACTCCTAAAAA \\
\hline & gDNA & Anti-sense & 5' CCTCTCTATGGGCAGTCGGTGATTCTGTGAAAACTTGAGGTTAAAAA \\
\hline
\end{tabular}


Alternatively, it can be assumed that one allele is derived from a distribution with an inflated mean solely due to experimental error (i.e., under the null hypothesis of no $\mathrm{AEI})$. In this case, we have that the means of the transcript reads satisfy either $\lambda_{G}=(1+\delta) \lambda_{T}$ or $(1+\delta) \lambda_{G}=\lambda_{T}$. Here, the probability parameter, $\mathrm{p}$, for the count probability in the AEI test becomes $\frac{1+\delta}{2+\delta}$ or $\frac{1}{2+\delta}$, respectively. For our gDNA data, we have a maximum $8.5 \%$ increase of one allele over the other and chose to conservatively assume a $20 \%$ mean increase (i.e., $\delta=0.2$ ). We then calculate the AEI test $\mathrm{p}$-value from the lesser-significant test of $H_{0}: p=\frac{1+\delta}{2+\delta}$ and $p=\frac{1}{2+\delta}$.

\section{Genotype association with AD risk}

The Mayo Clinic dataset has been described previously [31,32]. Briefly, the Mayo Clinic dataset contained 1789 cases and 2529 non-ADs collected from six centers from the US and Europe as described [32]. Direct genotyping of rs3851179 and rs588076 was performed using a TaqMan SNP genotyping assay in an ABI PRISM 7900HT Sequence Detection System with 384-well block module from Applied Biosystems (California, USA). First-pass genotype cluster calling was analyzed using the SDS software version 2.2.3 (Applied Biosystems, California, USA). Variants passed Hardy-Weinburg $(\mathrm{P}>0.05)$ and minor allele frequencies are consistent with public databases (EVS, HapMap, 1000G). Association testing for rs3851179, with and without rs588076, was carried out in PLINK [33] by using an additive logistic regression model corrected for appropriate covariates; diagnosis age, $A P O E$ 4, $A P O E$ 2, sex and contributing center.

\section{Additional file}

Additional file 1: Table S1. PICALM AEI analysis of AD40 shows significant unequal rs76719109T to $\mathrm{G}$ allele ratios.

\section{Competing interests}

The authors declare that they have no competing interests.

\section{Authors' contributions}

IP carried out the molecular genetic AEI studies and analysis and helped to draft the manuscript. CM and SG evaluated the SNP's association with AD risk. DWF participated in the design of the study and performed the statistical analysis. SE helped design the study and draft the manuscript. All authors read and approved the final manuscript.

\section{Acknowledgements}

This work is funded by National Institutes of Health [P01-AGO30128 and R01-AG045775 (SE), P30-AG028383, R25GM093044 and K25-AG043546 (DWF)] and Alzheimer's Research UK US travel fellowship (CWM).

\section{Author details}

${ }^{1}$ Departments of Physiology, Sanders-Brown Center on Aging, University of Kentucky, 800 S. Limestone St., Lexington, KY 40536, USA. ²Department of Neuroscience, Mayo Clinic Jacksonville, Jacksonville, FL 32224, USA. ${ }^{3}$ Biostatistics, Sanders-Brown Center on Aging, University of Kentucky, 800 S. Limestone St., Lexington, KY 40536, USA.
Received: 24 July 2014 Accepted: 21 August 2014

Published: 29 August 2014

\section{References}

1. Baig S, Joseph SA, Tayler H, Abraham R, Owen MJ, Williams J, Kehoe PG, Love S: Distribution and expression of picalm in Alzheimer disease. J Neuropathol Exp Neurol 2010, 69:1071-1077.

2. Parikh I, Fardo DW, Estus S: Genetics of PICALM expression and Alzheimer's disease. PLoS One 2014, 9:e91242.

3. Xiao Q, Gil SC, Yan P, Wang Y, Han S, Gonzales E, Perez R, Cirrito JR, Lee JM: Role of phosphatidylinositol clathrin assembly lymphoid-myeloid leukemia (PICALM) in intracellular amyloid precursor protein (APP) processing and amyloid plaque pathogenesis. J Biol Chem 2012, 287:21279-21289.

4. D'Angelo F, Vignaud H, Di Martino J, Salin B, Devin A, Cullin C, Marchal C: A yeast model for amyloid-beta aggregation exemplifies the role of membrane trafficking and PICALM in cytotoxicity. Dis Model Mech 2013, 6:206-216.

5. Kanatsu K, Morohashi Y, Suzuki M, Kuroda H, Watanabe T, Tomita T, Iwatsubo T: Decreased CALM expression reduces Abeta42 to total Abeta ratio through clathrin-mediated endocytosis of gamma-secretase. Nat Commun 2014, 5:3386.

6. Harold D, Abraham R, Hollingworth P, Sims R, Gerrish A, Hamshere ML, Pahwa JS, Moskvina V, Dowzell K, Williams A, Jones N, Thomas C, Stretton A, Morgan AR, Lovestone S, Powell J, Proitsi P, Lupton MK, Brayne C, Rubinsztein DC, Gill M, Lawlor B, Lynch A, Morgan K, Brown KS, Passmore PA, Craig D, McGuinness B, Todd S, Holmes C, et al: Genome-wide association study identifies variants at CLU and PICALM associated with Alzheimer's disease. Nat Genet 2009, 41:1088-1093.

7. Jun G, Naj AC, Beecham GW, Wang LS, Buros J, Gallins PJ, Buxbaum JD, Ertekin-Taner N, Fallin MD, Friedland R, Inzelberg R, Kramer P, Rogaeva E, St George-Hyslop P, Cantwell LB, Dombroski BA, Saykin AJ, Reiman EM, Bennett DA, Morris JC, Lunetta KL, Martin ER, Montine TJ, Goate AM, Blacker D, Tsuang DW, Beekly D, Cupples LA, Hakonarson H, Kukull W, et al: Meta-analysis confirms CR1, CLU, and PICALM as alzheimer disease risk loci and reveals interactions with APOE genotypes. Arch Neurol 2010, 67:1473-1484

8. Lambert JC, Heath S, Even G, Campion D, Sleegers K, Hiltunen M, Combarros O, Zelenika D, Bullido MJ, Tavernier B, Letenneur L, Bettens K, Berr C, Pasquier F, Fievet N, Barberger-Gateau P, Engelborghs S, De Deyn P, Mateo I, Franck A, Helisalmi S, Porcellini E, Hanon O, de Pancorbo MM, Lendon C, Dufouil C, Jaillard C, Leveillard T, Alvarez V, Bosco P, et al: Genome-wide association study identifies variants at CLU and CR1 associated with Alzheimer's disease. Nat Genet 2009, 41:1094-1099.

9. Seshadri S, Fitzpatrick AL, Ikram MA, DeStefano AL, Gudnason V, Boada M, Bis JC, Smith AV, Carassquillo MM, Lambert JC, Harold D, Schrijvers EM, Ramirez-Lorca R, Debette S, Longstreth WT Jr, Janssens AC, Pankratz VS, Dartigues JF, Hollingworth P, Aspelund T, Hernandez I, Beiser A, Kuller LH, Koudstaal PJ, Dickson DW, Tzourio C, Abraham R, Antunez C, Du Y, Rotter Jl, et al: Genome-wide analysis of genetic loci associated with Alzheimer disease. JAMA 2010, 303:1832-1840.

10. Pedraza O, Allen M, Jennette $K$, Carrasquillo M, Crook J, Serie D, Pankratz VS, Palusak R, Nguyen T, Malphrus K, Ma L, Bisceglio G, Roberts RO, Lucas JA, Ivnik RJ, Smith GE, Graff-Radford NR, Petersen RC, Younkin SG, Ertekin-Taner N: Evaluation of memory endophenotypes for association with CLU, CR1, and PICALM variants in black and white subjects. Alzheimers Dement 2014, 10:205-213.

11. Liu G, Zhang S, Cai Z, Ma G, Zhang L, Jiang Y, Feng R, Liao M, Chen Z, Zhao B, Li K: PICALM gene rs3851179 polymorphism contributes to Alzheimer's disease in an Asian population. Neuromolecular Med 2013, 15:384-388.

12. Miyashita A, Koike A, Jun G, Wang LS, Takahashi S, Matsubara E, Kawarabayashi T, Shoji M, Tomita N, Arai H, Asada T, Harigaya Y, Ikeda M, Amari M, Hanyu H, Higuchi S, Ikeuchi T, Nishizawa M, Suga M, Kawase Y, Akatsu H, Kosaka K, Yamamoto T, Imagawa M, Hamaguchi T, Yamada M, Moriaha T, Takeda M, Takao T, Nakata $\mathrm{K}$, et al: SORL1 is genetically associated with late-onset Alzheimer's disease in Japanese, Koreans and Caucasians. PloS one 2013, 8:e58618.

13. Yu JT, Song JH, Ma T, Zhang W, Yu NN, Xuan SY, Tan L: Genetic association of PICALM polymorphisms with Alzheimer's disease in Han Chinese. J Neurol Sci 2011, 300:78-80.

14. Li HL, Shi SS, Guo QH, Ni W, Dong Y, Liu Y, Sun YM, Bei W, Lu SJ, Hong Z, WU ZY: PICALM and CR1 variants are not associated with sporadic Alzheimer's disease in Chinese patients. J Alzheimers Dis 2011, 25:111-117. 
15. Chen LH, Kao PY, Fan YH, Ho DT, Chan CS, Yik PY, Ha JC, Chu LW, Song YQ: Polymorphisms of CR1, CLU and PICALM confer susceptibility of Alzheimer's disease in a southern Chinese population. Neurobiol Aging 2012, 33:210. e211-217.

16. Mondal AK, Sharma NK, Elbein SC, Das SK: Allelic expression imbalance screening of genes in chromosome 1q21-24 region to identify functional variants for Type 2 diabetes susceptibility. Physiol Genomics 2013, 45:509-520.

17. Jones BL, Swallow DM: The impact of cis-acting polymorphisms on the human phenotype. The HUGO journal 2011, 5:13-23.

18. Pham MH, Bonello GB, Castiblanco J, Le T, Sigala J, He W, Mummidi S: The rs1024611 regulatory region polymorphism is associated with CCL2 allelic expression imbalance. PLoS One 2012, 7:e49498.

19. Jentarra GM, Rice SG, Olfers S, Saffen D, Narayanan V: Evidence for population variation in TSC1 and TSC2 gene expression. BMC Med Genet 2011, 12:29.

20. Smith RM, Webb A, Papp AC, Newman LC, Handelman SK, Suhy A, Mascarenhas R, Oberdick J, Sadee W: Whole transcriptome RNA-Seq allelic expression in human brain. BMC Genomics 2013, 14:571.

21. Kamide K, Asayama K, Katsuya T, Ohkubo T, Hirose T, Inoue R, Metoki H, Kikuya M, Obara T, Hanada H, Thijs L, Kuznetsova T, Noguchi Y, Sugimoto K, Ohishi M, Morimoto S, Nakahashi T, Takiuchi S, Ishimitsu T, Tsuchihashi T, Soma M, Higaki J, Matsuura H, Shinagawa T, Sasaguri T, Miki T, Takeda K, Shimamoto K, Ueno M, Hosomi N, et al: Genome-wide response to antihypertensive medication using home blood pressure measurements: a pilot study nested within the HOMED-BP study. Pharmacogenomics 2013, 14:1709-1721

22. Xu X, Wang H, Zhu M, Sun Y, Tao Y, He Q, Wang J, Chen L, Saffen D: Nextgeneration DNA sequencing-based assay for measuring allelic expression imbalance (AEI) of candidate neuropsychiatric disorder genes in human brain. BMC Genomics 2011, 12:518.

23. Johnson AD, Handsaker RE, Pulit SL, Nizzari MM, O'Donnell CJ, de Bakker PI: SNAP: a web-based tool for identification and annotation of proxy SNPs using HapMap. Bioinformatics 2008, 24:2938-2939.

24. Sherry ST, Ward MH, Kholodov M, Baker J, Phan L, Smigielski EM, Sirotkin K: dbSNP: the NCBI database of genetic variation. Nucleic Acids Res 2001, 29:308-311

25. Scotland $\mathrm{PB}$, Heath $\mathrm{JL}$, Conway $\mathrm{AE}$, Porter NB, Armstrong MB, Walker JA, Klebig ML, Lavau CP, Wechsler DS: The PICALM protein plays a key role in iron homeostasis and cell proliferation. PLoS One 2012, 7:e44252.

26. Malik M, Simpson JF, Parikh I, Wilfred BR, Fardo DW, Nelson PT, Estus S: CD33 Alzheimer's Risk-Altering Polymorphism, CD33 Expression, and Exon 2 Splicing. J Neurosci 2013, 33:13320-13325.

27. Ling IF, Bhongsatiern J, Simpson JF, Fardo DW, Estus S: Genetics of clusterin isoform expression and Alzheimer's disease risk. PLoS One 2012, 7:e33923.

28. Fleige S, Pfaffl MW: RNA integrity and the effect on the real-time qRT-PCR performance. Mol Aspects Med 2006, 27:126-139.

29. Thomas M, William ML, Mattieu M, Rishi N, Bert O, Miguel P, Bethan P, Emily P, Harpreet Singh R, Graham RS R, Magali R, Michael S, Daniel S, Daniel S, Kieron T, Anja T, Stephen T, Simon W, Wilder SP, Aken BL, Ewan B, Fiona C, Ian D, Jennifer H, Javier H, Tim JP H, Nathan J, Rhoda K, Anne P, Giulietta S, et al: Ensembl 2014. Nucleic Acids Research 2014, 42(Database issue):D749-D755.

30. Jiang $\mathrm{H}$, Wong WH: Statistical inferences for isoform expression in RNA-Seq. Bioinformatics 2009, 25:1026-1032.

31. Ridge PG, Mukherjee S, Crane PK, Kauwe JS, Alzheimer's Disease Genetics C: Alzheimer's disease: analyzing the missing heritability. PLoS One 2013, 8:e79771.

32. Naj AC, Jun G, Beecham GW, Wang LS, Vardarajan BN, Buros J, Gallins PJ, Buxbaum JD, Janvik GP, Crane PK, Larson EB, Bird TD, Boeve BF, Graff-Radford NR, De Jager PL, Evans D, Schneider JA, Carrasquillo MM, Ertekin-Taner N, Younkin SG, Cruchaga C, Kauwe JS, Nowotny P, Kramer P, Hardy J, Huentelman MJ, Myers AJ, Barmada MM, Demirci FY, Baldwin CT, et al: Common variants at MS4A4/MS4A6E, CD2AP, CD33 and EPHA1 are associated with late-onset Alzheimer's disease. Nat Genet 2011, 43:436-441.

33. Purcell $S$, Neale B, Todd-Brown $K$, Thomas L, Ferreira MA, Bender D, Maller J, Sklar P, de Bakker PI, Daly MJ, Sham PC: PLINK: a tool set for whole-genome association and population-based linkage analyses. Am J Hum Genet 2007, 81:559-575.

doi:10.1186/1750-1326-9-32

Cite this article as: Parikh et al: An intronic PICALM polymorphism, rs588076, is associated with allelic expression of a PICALM isoform. Molecular Neurodegeneration 2014 9:32.

\section{Submit your next manuscript to BioMed Central and take full advantage of:}

- Convenient online submission

- Thorough peer review

- No space constraints or color figure charges

- Immediate publication on acceptance

- Inclusion in PubMed, CAS, Scopus and Google Scholar

- Research which is freely available for redistribution 